SCIENTIFIC LETTER

\title{
Green tea reverses endothelial dysfunction in healthy smokers
}

\author{
N Nagaya, H Yamamoto, M Uematsu, T ltoh, K Nakagawa, T Miyazawa, K Kangawa, \\ K Miyatake
}

Heart 2004;90:1485-1486. doi: 10.1136/hrt.2003.026740

G reen tea is popular in Asia, including Japan, although it is rarely consumed by western people. It contains large amounts of catechins which are potent free radical scavengers and therefore has an antioxidant effect. ${ }^{1}$ Oxidative stress participates in the pathogenesis of many cardiovascular diseases. Recently, black tea consumption has been shown to reverse endothelial dysfunction in patients with coronary artery disease through its antioxidant effects. ${ }^{2}$ However, the number of catechins in non-fermented tea (green tea) is higher than that in fermented tea (black tea). ${ }^{1}$ These findings raise the possibility that green tea acts as a potent antioxidant.

Smoking is a well recognised risk factor for cardiovascular diseases. Free radicals in cigarette smoke are responsible for endothelial dysfunction, leading to atherosclerosis. ${ }^{3}$ Earlier studies have shown that the flavonoid components of red wine and purple grape juice, which are powerful antioxidants, reverse endothelial dysfunction and reduce cardiovascular risk. However, any beneficial effect of green tea on vascular function in smokers remains unknown. Accordingly, the purpose of this study was to investigate whether green tea consumption reverses endothelial dysfunction in association with improvement of oxidative stress in healthy smokers.

\section{METHODS}

We studied 20 healthy male smokers (mean (SEM) age 33 (1) years, body mass index $22.9(0.9) \mathrm{kg} / \mathrm{m}^{2}$, smoking 15.5 (2.1) packs/year). They were randomised to consume green tea or hot water in a crossover design. All subjects gave written informed consent and the study was approved by the ethics committee of the National Cardiovascular Center. Subjects were studied on two separate days, at least one week apart, at 8:30 in the morning after they had fasted for at least 12 hours. Subjects did not smoke on the morning of the study. At baseline and two hours after consumption of $400 \mathrm{ml}$ green tea or hot water, we measured the response of forearm blood flow (FBF) to reactive hyperaemia, an index of endothelium dependent vasodilatation, and to sublingual administration of glyceryl trinitrate (GTN), an index of endothelium independent vasodilatation. To induce reactive hyperaemia, FBF was occluded by inflating a cuff around the left upper arm to a pressure of $280 \mathrm{~mm}$ ?Hg for five minutes. After release of the ischaemic cuff occlusion, FBF was measured for three minutes. After FBF returned to the baseline in a 15 minute recovery period, $0.3 \mathrm{mg}$ GTN was administered sublingually and FBF was measured for five minutes. Seven healthy volunteers (mean age 32 (2) years), who had not smoked for more than one year, served as age matched non-smoking controls.

FBF was measured using a mercury filled Silastic straingauge plethysmograph (EC-5R, DE Hokanson Inc, Washington, USA), as described previously. ${ }^{1}$ FBF was expressed in $\mathrm{ml} / \mathrm{min} / 100 \mathrm{ml}$ of forearm tissue volume.
Blood and urine samples were repeatedly obtained for measurement of plasma catechin and urine 8-iso-prostaglandinF $2 \alpha$ (8-iso-PGF $2 \alpha$ ), an index of oxidative stress. ${ }^{4}$ Numerical values were expressed as mean (SEM). Comparisons of the time course of parameters between the two groups were made by two way analysis of variance (ANOVA) for repeated measures, followed by Scheffe's multiple comparison test.

\section{RESULTS}

Green tea contained very large amounts of catechins $(173.1 \mathrm{mg} / \mathrm{dl})$, including epigallocatechin gallate $(61.8 \mathrm{mg} /$ dl), which have potent antioxidant effects. The content of epigallocatechin gallate in green tea was 10 -fold higher than that in black tea. Unlike black tea, green tea also contained ascorbic acid $(3.0 \mathrm{mg} / \mathrm{dl})$. Plasma epigallocatechin gallate concentration was notably increased two hours after a single consumption of green tea (3 (1) pmol/ml to 694 (81) pmol/ $\mathrm{ml}, \mathrm{p}<0.001)$. Green tea consumption significantly decreased the urinary concentration of 8 -iso-PGF $2 \alpha$, an index of oxidative stress $(541$ (188) $\mathrm{pg} / \mathrm{mg}$ creatinine to 396 (136) pg/mg creatinine, p < 0.05). Green tea had no significant effect on systolic and diastolic blood pressure, heart rate, fasting plasma lipid, or glucose concentration. These parameters remained unchanged after hot water consumption.

During reactive hyperaemia in healthy smokers, FBF was significantly less than that in age matched non-smoking controls (maximum FBF, 16.9 (1.1) $\mathrm{ml} / \mathrm{min} / 100 \mathrm{ml}$ tissue $v$ $29.3(2.1) \mathrm{ml} / \mathrm{min} / 100 \mathrm{ml}$ tissue, $\mathrm{p}<0.01$ ), suggesting the presence of endothelial dysfunction in healthy smokers. Time course curves of FBF during reactive hyperaemia were similar before consumption of green tea and hot water. When the results of subjects randomised to receive green tea first and hot water first were combined, green tea consumption significantly increased $\mathrm{FBF}$ during reactive hyperaemia (maximum FBF, 16.9 (1.1) $\mathrm{ml} / \mathrm{min} / 100 \mathrm{ml}$ tissue to 21.3 (1.3) $\mathrm{ml} / \mathrm{min} / 100 \mathrm{ml}$ tissue, $\mathrm{p}<0.001$, fig $\mathrm{l}$ ).

These results suggest that green tea consumption improved endothelium dependent vasodilatation in smokers. In contrast, green tea had no effect on the increase in FBF after sublingual administration of GTN, an index of endothelium independent vasodilatation (maximum FBF, 16.9 (1.1) ml/ $\mathrm{min} / 100 \mathrm{ml}$ tissue to $21.3 \quad(1.3) \mathrm{ml} / \mathrm{min} / 100 \mathrm{ml}$ tissue, $\mathrm{p}=\mathrm{NS}$ ). These parameters remained unchanged after hot water consumption.

\section{DISCUSSION}

The present study demonstrated that, compared with black tea, green tea contained extremely large amounts of catechins including epigallocatechin gallate. We also showed that, unlike black tea, green tea contained ascorbic acid, which also has antioxidant effects. These results suggest that green tea may act as a potent antioxidant. 

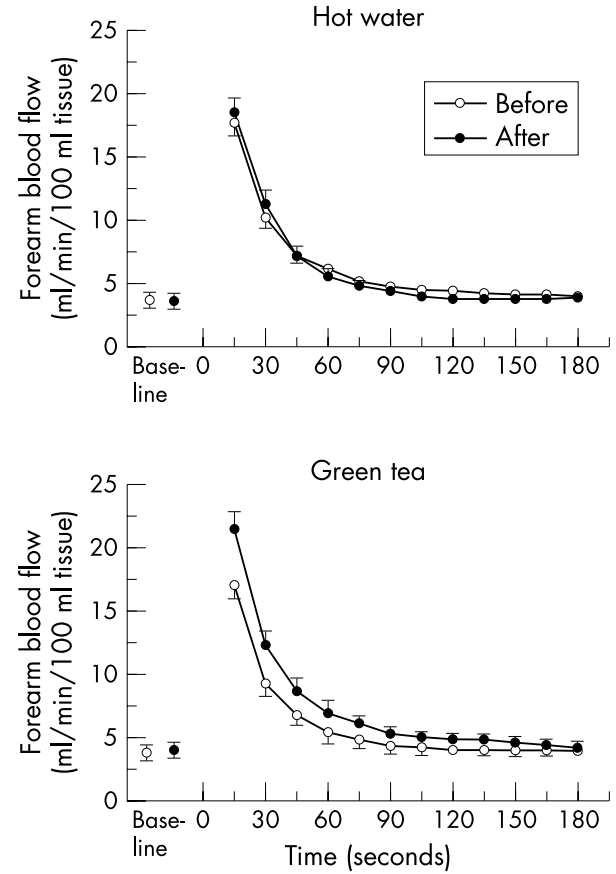

Figure 1 Forearm blood flow (FBF) at rest and during reactive hyperaemia before (open circles) and after (solid circles) consumption of hot water (upper panel) or green tea (lower panel). Green tea consumption augmented FBF during reactive hyperaemia $(p<0.001)$ whereas hot water consumption did not significantly alter it.

Cigarette smoke has been reported to contain nicotine and large amounts of free radicals, such as superoxide anion and hydroxyradicals. ${ }^{3}$ Free radicals in cigarette smoke may not only degrade nitric oxide released from the endothelium, but also produce highly reactive intermediates, resulting in endothelial injury. In the present study, a single consumption of green tea notably increased plasma epigallocatechin gallate concentration and significantly decreased urinary 8-isoPGF2 $\alpha$, a specific and stable product of lipid peroxidation, in healthy smokers. Another study has shown that green tea decreases oxidative DNA damage (measured through 8-OHdG (8-hydroxy-2'-deoxyguanosine) in smokers. ${ }^{5}$ These results suggest that green tea consumption attenuates oxidative stress in healthy smokers, at least in part, through the antioxidant effects of catechins. Ascorbic acid included in green tea may also play a role in the regulation of oxidative stress. As expected, endothelium dependent vasodilatation was impaired in healthy smokers, consistent with many previous studies.

In the present study, a single consumption of green tea significantly increased FBF during reactive hyperaemia in smokers. In contrast, green tea had no effect on FBF at rest or GTN induced vasodilatation. These results suggest that green tea consumption reverses endothelial dysfunction in healthy smokers, possibly through its antioxidant effect. Earlier studies have demonstrated that endothelial dysfunction is important in the pathogenesis and clinical manifestation of cardiovascular disease. Thus, green tea consumption may be beneficial for the prevention and treatment of atherosclerotic vascular disease. Further prospective, randomised studies of green tea consumption are necessary to examine whether green tea consumption reduces the risk of cardiovascular events and mortality.

\section{ACKNOWLEDGEMENTS}

This work was supported by the Promotion of Fundamental Studies in Health Science of the Organization for Pharmaceutical Safety and Research (OPSR) of Japan.

\section{Authors' affiliations}

N Nagaya, H Yamamoto, T Itoh, K Miyatake, Department of Internal Medicine, National Cardiovascular Center, Osaka, Japan M Uematsu, Cardiovascular Division, Kansai Rosai Hospital, Hyogo, Japan

K Nakagawa, T Miyazawa, Laboratory of Biodynamic Chemistry, Tohoku University Graduate School of Life Science and Agriculture, Sendai, Japan

K Kangawa, Department of Biochemistry, National Cardiovascular Center Research Institute, Osaka, Japan

Correspondence to: Dr Noritoshi Nagaya, Department of Internal Medicine, National Cardiovascular Center, 5-7-1 Fujishirodai, Suita, Osaka 565-8565, Japan; nagayann@hsp.ncvc.go.jp

Accepted 26 February 2004

\section{REFERENCES}

1 Wiseman H, Plitzanopoulou P, O'Reilly J. Antioxidant properties of ethanolic and aqueous extracts of green tea compared to black tea. Biochem Soc Trans 1996;24:390S.

2 Duffy SJ, Keaney JF Jr, Holbrook M, et al. Short- and long-term black tea consumption reverses endothelial dysfunction in patients with coronary artery disease. Circulation 2001;104:151-6.

3 Benowitz N. Drug therapy: Pharmacological aspects of cigarette smoking and nicotine addiction. N Engl J Med 1988;319:1318-30.

4 Artaud-Wild SM, Connor SL, Sexton G, et al. Differences in coronary mortality can be explained by differences in cholesterol and saturated fat intakes in 40 countries but not in France and Finland: a paradox. Circulation 1993;88:2771-9.

5 Klaunig JE, Xu Y, Han $\mathrm{C}$, et al. The effect of tea consumption on oxidative stress in smokers and nonsmokers. Proc Soc Exp Biol Med 1999;220:249-54. 